\title{
Strong planar subsystem symmetry-protected topological phases and their dual fracton orders
}

\author{
Trithep Devakul $\odot$ \\ Department of Physics, Princeton University, Princeton, New Jersey 08540, USA \\ Wilbur Shirley \\ Department of Physics and Institute for Quantum Information and Matter, California Institute of Technology, \\ Pasadena, California 91125, USA \\ Juven Wang $\odot$ \\ Center of Mathematical Sciences and Applications, Harvard University, Cambridge, Massachusetts 02138, USA \\ and School of Natural Sciences, Institute for Advanced Study, Einstein Drive, Princeton, New Jersey 08540, USA
}

(Received 28 November 2019; revised manuscript received 7 February 2020; accepted 10 February 2020; published 12 March 2020)

\begin{abstract}
We classify subsystem symmetry-protected topological (SSPT) phases in $3+1$ dimensions (3+1D) protected by planar subsystem symmetries: short-range entangled phases which are dual to long-range entangled Abelian fracton topological orders via a generalized "gauging" duality. We distinguish between weak SSPTs, which can be constructed by stacking 2+1D SPTs, and strong SSPTs, which cannot. We identify signatures of strong phases, and show by explicit construction that such phases exist. A classification of strong phases is presented for an arbitrary finite Abelian group. Finally, we show that fracton orders realizable via $p$-string condensation are dual to weak SSPTs, while those dual to strong SSPTs exhibit statistical interactions prohibiting such a realization.
\end{abstract}

DOI: 10.1103/PhysRevResearch.2.012059

Introduction. Global symmetries, such as the $\mathbb{Z}_{2}$ spin-flip symmetry of the Ising model, act throughout the bulk of a system. Recently, there has been an emerging interest in symmetries that act on only part of a system. These include higher-form symmetries which act on deformable lowerdimensional manifolds of a system [1], as well as subsystem symmetries [2-4], which act on rigid lower-dimensional subsystems. It has also been realized that such subsystem symmetries may protect nontrivial symmetry-protected topological (SPT) phases [5-8]: gapped, disordered, short-range entangled phases which cannot be adiabatically connected to the trivial disordered phase in the presence of symmetry, but can be if the symmetry is not enforced. Examples of subsystem symmetries include those which act along linear [9,10], planar $[11,12]$, or even fractal [13-18] subsystems. Such phases have been aptly named subsystem SPT (SSPT) phases, and this Rapid Communication concerns their classification.

In $2+1$ dimensions $(2+1 \mathrm{D})$, such systems have gained interest due to the discovery that nontrivial SSPT phases may serve as a resource for universal measurement-based quantum computation (MBQC) [19-24] and also due to their unusual patterns of quantum entanglement [10,25-28]. In attempting

Published by the American Physical Society under the terms of the Creative Commons Attribution 4.0 International license. Further distribution of this work must maintain attribution to the author(s) and the published article's title, journal citation, and DOI. to classify $2+1 \mathrm{D}$ linear SSPTs, one is faced with the issue that there are uncountably infinitely many distinct phases. This is due to the presence of weak phases: SSPT phases which can be constructed by stacking (a process which we will define) $1+1 \mathrm{D}$ SPTs along the subsystems, whose nontrivialities are simply a manifestation of lower-dimensional physics. Reference [10] defined an equivalence relation between phases wherein two phases that differ by stacking 1+1D SPTs belong to the same equivalence class. Phases not in the trivial equivalence class are, by definition, strong SSPTs. It was found that there are a small number of equivalence classes, which provided a sensible classification for the uncountably infinite phases. In contrast, note that for $2+1 \mathrm{D}$ fractal SSPTs, weak phases do not exist and the number of phases is countably infinite [15]. This Rapid Communication is the natural extension of Ref. [10] to planar symmetries in 3+1D (henceforth simply 3D).

Systems with planar subsystem symmetries have also received intense interest recently due to the discovery that, under a generalized "gauging" duality [11,16,29], they map onto long-range entangled models exhibiting fracton topological order [13,30-48]. An example of such a system is the plaquette Ising model $[11,49,50]$, whose paramagnetic phase is dual to the $\mathrm{X}$-cube model of fracton topological order [11]. Fracton phases are characterized by a subextensive topological ground-state degeneracy growing exponentially with $L$, and quasiparticle excitations with limited mobility. The classification of such fracton phases is an active topic of research [51-59]. In this Rapid Communication, we focus on 
the classification of SSPT phases which are dual to Abelian fracton phases, thus also providing a useful means of categorizing such fracton phases.

The brief history of 3D planar SSPT phases begins with Ref. [12], which constructed a nontrivial 3D planar SSPT model. However, it was later discovered that its fracton dual belonged to the same foliated fracton phase as the X-cube model [60], implying that it is weak. More recently, fracton phases were constructed in Ref. [58] which possess "twisted" foliated fracton orders, raising the question as to the nature of their SSPT duals. We find that these phases, too, are weak. This prompts the question: Do any strong planar SSPTs exist? We answer this in the affirmative. We explicitly construct strong SSPT phases, which are dual to strong fracton phases with unusual braiding statistics that cannot be obtained by coupling 2D theories. In this sense these statistical interactions are "intrinsically" three-dimensional.

We will first show how to construct weak 3D planar SSPT phases via a stacking process of 2D SPTs. We then ask whether there are SSPT phases which cannot be realized by this process. We identify mechanisms by which an SSPT may be strong, leading to a classification of such phases, and construct exactly solvable, zero-correlation length models realizing these phases. In the fracton dual picture, this construction corresponds to one in which 2D topological orders are stacked onto and strongly coupled to an existing fracton model [58]. The duals of our strong SSPTs are fracton phases which cannot be attained via such a procedure, also implying that they cannot be realized by a $p$-string condensation transition [46,61], as we will show.

Planar subsystem symmetries. Throughout we will consider a system with degrees of freedom on each site of a cubic lattice. Each site $\mathbf{r}$ transforms under the finite Abelian onsite symmetry group $G$ under a unitary representation $u_{\mathbf{r}}(g)$, where $g \in G$. An $x y$ planar symmetry acting on plane $z$ acts as $S^{x y}(z ; g)=\prod_{x, y} u_{\mathbf{r}=(x, y, z)}(g)$ for $g \in G$. Similarly, we may define $S^{y z}(x ; g)$ and $S^{z x}(y ; g)$, which act on $y z$ and $z x$ planes, respectively. Importantly, individual sites transform under the same on-site representation regardless of the orientation of the planar symmetry-there is therefore a redundancy: The product of all $x y$ symmetries is identical to the product of all $y z$ or all $z x$ symmetries. We will refer to models which respect only one orientation of planar symmetry as 1-foliated, those with two as 2-foliated, and those with all three as 3-foliated. To construct explicit models, we choose the on-site degrees of freedom to be $G$-valued, $\left|g_{\mathbf{r}}\right\rangle$, which transform under the on-site symmetry as $u_{\mathbf{r}}(g)\left|g_{\mathbf{r}}\right\rangle=\left|g g_{\mathbf{r}}\right\rangle$.

Construction of weak SSPT phases. It is possible to construct nontrivial SSPT phases from known 2D global SPTs, as we will show in this section. Phases obtained in this way are "weak," by definition, whose nontrivial properties are in some sense a manifestation of lower-dimensional physics. We emphasize here that we do not assume any translation invariance in our system. Hence, our definition is different (but similar in spirit) to weak crystalline SPTs with global symmetries, which are stacks of lower-dimensional SPTs protected by translation symmetry.

First, we briefly review the group cohomological classification of 2D bosonic SPTs with global symmetry group $G[7,62]$. For the purpose of being self-contained, we also include a more detailed review in the Supplemental Material [63]. The classification of such phases [7,64] is given by the third cohomology group $H^{3}[G, U(1)]$. For simplicity, we may consider $G=\left(\mathbb{Z}_{N}\right)^{n}$, in which case an element of $H^{3}[G, U(1)]$ is specified by integers, $p_{\mathrm{I}}^{i}(i \in[1, n]), p_{\mathrm{II}}^{i j}(i<$ $j)$, and $p_{\mathrm{III}}^{i j k}(i<j<k)$, all modulo $N$, called type-I, -II, and -III cocycles, respectively. We will specify $p_{\mathrm{I}}^{i}$ and $p_{\mathrm{II}}^{i j}$ compactly in a single symmetric $n \times n$ integer matrix $\mathbf{M}$ with $M_{i i}=2 p_{\mathrm{I}}^{i}$ and $M_{i j}=M_{j i}=p_{\mathrm{II}}^{i j}$. Upon gauging the global symmetries of a 2D SPT, one obtains a topologically ordered system with fractional quasiparticles carrying gauge charge or flux (or both). Nontrivial type-III cocycles give rise to nonAbelian topological order $[65,66]$, which we will not consider here. A generating set of quasiparticles are the "electric" excitations (gauge charges) $\left\{e_{i}\right\}$ and "magnetic" excitations (gauge fluxes) $\left\{m_{i}\right\}$. Each $e_{i}$ has an $e^{2 \pi i / N}$ braiding statistic with $m_{i}$ and trivial statistics with all other generators. The elements of $\mathbf{M}$ characterize the self- and mutual statistics of gauge flux excitations [62]. In particular, the type-I cocycles give rise to a self-exchange statistic $e^{\pi i M_{i i} / N^{2}}$ of the gauge flux $m_{i}$, and type-II cocycles lead to a mutual braiding statistic of $e^{2 \pi i M_{i j} / N^{2}}$ between $m_{i}$ and $m_{j}$. Note that these phases are only well-defined modulo $e^{2 \pi i / N}$, since flux is only well defined up to attachment of charge, $m_{i} \rightarrow m_{i} e_{j}$. Finally, we note that Abelian topological orders in $2 \mathrm{D}$ can all be described by K-matrix Chern-Simons theories [67,68]. The topological orders we have discussed have a $2 n \times 2 n \mathbf{K}$-matrix description with

$$
\mathbf{K}=N\left[\begin{array}{cc}
-\frac{1}{N} \mathbf{M} & \mathbf{1} \\
\mathbf{1} & \mathbf{0}
\end{array}\right], \quad \mathbf{K}^{-1}=\frac{1}{N}\left[\begin{array}{cc}
\mathbf{0} & \mathbf{1} \\
\mathbf{1} & \frac{1}{N} \mathbf{M}
\end{array}\right],
$$

where the indices labeling quasiparticles are ordered as $\left\{e_{1}, \ldots, e_{n}, m_{1}, \ldots, m_{n}\right\}$. Quasiparticles are described by an integer vector $\ell$ in this basis, and have a self-exchange statistic $e^{\pi i \ell^{T} \cdot \mathbf{K}^{-1} \cdot \ell}$ and mutual braiding statistics $e^{2 \pi i \ell_{1}^{T} \cdot \mathbf{K}^{-1} \cdot \ell_{2}}$.

It is always possible to view a 3D planar SSPT as a quasi-2D system in the $x y$ plane with a subextensively large symmetry group $G^{L}$ by compactifying the $z$ direction. We may then proceed to compute its classification in terms of $H^{3}\left[G^{L}, U(1)\right]$, which is characterized by a subextensively large $\mathbf{M}$ matrix. We note that it is possible to define $\mathbf{M}$ matrices corresponding to $y z$ or $z x$ as well, but for reasons that will become clear we will always consider the $x y$ symmetries only. It is useful to introduce a graphical notation for $\mathbf{M}$, which is used in Fig. 1. The $\alpha$ th generator of $G$ in a plane $z$ is denoted by a vertex $a_{i=(\alpha, z)}$. Two vertices $i$ and $j$ are connected by an undirected edge with weight $M_{i j}$, and a vertex $i$ is connected to itself via a self-loop with weight $M_{i i} / 2$, where weights are defined modulo $N$

Consider the 2D global symmetry group $G_{2 \mathrm{D}}=G^{K}$ for an integer $K$. For an appropriate choice of the pure phase function $f_{2 \mathrm{D}}$, the wave function $|\psi\rangle_{2 \mathrm{D}}=\sum_{\left\{g_{\mathbf{r}}\right\}} f_{2 \mathrm{D}}\left(\left\{g_{\mathbf{r}}\right\}\right)\left|\left\{g_{\mathbf{r}}\right\}\right\rangle_{2 \mathrm{D}}$ on a $2 \mathrm{D}$ square lattice is a zero-correlation length ground state of a commuting Hamiltonian with SPT order [63]. All phases in the group cohomology classification can be realized in this way $[66,69,70]$.

Suppose we start with the trivial disordered wave function $\left|\psi_{0}\right\rangle=\sum_{\left\{g_{\mathbf{r}}\right\}}\left|\left\{g_{\mathbf{r}}\right\}\right\rangle$ on the 3D cubic lattice. We can construct a nontrivial 1-foliated SSPT by identifying each factor of $G$ 


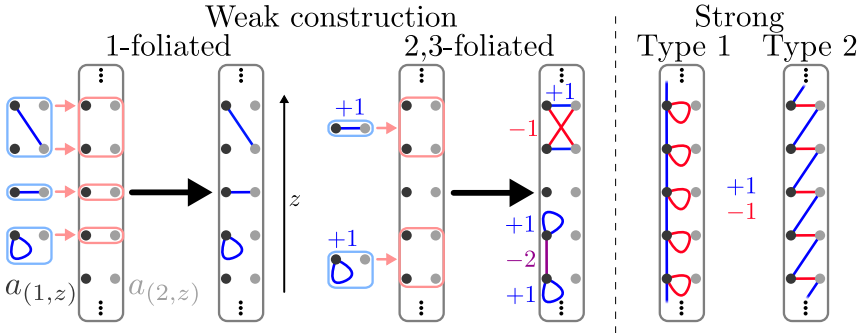

FIG. 1. Left: Examples of our construction of 1-foliated or weak 2- or 3-foliated models, for $G=\mathbb{Z}_{N} \times \mathbb{Z}_{N}$, in the graphical notation. 2D SPTs to be stacked are shown in the blue boxes, and the large arrow points to the resulting SSPT after stacking. The color of the edges connecting two vertices indicate its weight modulo $N$. Right: Examples of $\mathbf{M}$ matrices that cannot be obtained by stacking 2D phases onto 2- or 3-foliated models. The type-1 phase is only strong for even $N$, and type- 2 strong phases can only be realized for 2-foliated symmetries.

in $G_{2 \mathrm{D}}$ in the function $f_{2 \mathrm{D}}\left(\left\{g_{r}\right\}\right)$ with a planar $G$ symmetry in an arbitrary collection of planes $z_{1}, \ldots, z_{K}$ (where $z_{k}$ are all within some finite range to ensure locality). The wave function $|\psi\rangle_{1-\text { fol }}=U\left|\psi_{0}\right\rangle$ with $U=\sum_{\left\{g_{\mathbf{r}}\right\}} f_{2 \mathrm{D}}\left(\left\{g_{\mathbf{r}}\right\}_{r_{z} \in\left\{z_{k}\right\}}\right)\left|\left\{g_{\mathbf{r}}\right\}\right\rangle\left\langle\left\{g_{\mathbf{r}}\right\}\right|$ is the ground state of a 1-foliated 3D SSPT, which is nontrivial only near the planes $z_{k}$. We may then repeat this procedure arbitrarily many times, each time acting on the previous state with $U$ for different choices of $f_{2 \mathrm{D}}$ and $\left\{z_{k}\right\}$. We will call this procedure "stacking" the 2D SPT $|\psi\rangle_{2 \mathrm{D}}$ onto the planes $\left\{z_{k}\right\}$ of a 1-foliated SSPT.

More generally, we may define a stacking operation between two SSPTs in which the two systems, with on-site symmetry representations $u_{\mathbf{r}}^{(1)}(g)$ and $u_{\mathbf{r}}^{(2)}(g)$, are placed on top of each other to create a new SSPT with on-site representation $u_{\mathbf{r}}(g)=u_{\mathbf{r}}^{(1)}(g) \otimes u_{\mathbf{r}}^{(2)}(g)$. The group structure of the standard SPT classification is realized under such a stacking operation. Stacking a 2D SPT onto a 3D SSPT can be viewed as stacking two 3D SSPTs, in which the first is only nontrivial in the vicinity of a number of planes $\left\{z_{k}\right\}$. We define any phase realizable by stacking $2 \mathrm{D}$ SPTs in this way to be weak. In the case of our 1-foliated SSPT construction, each additional stacked 2D SPT simply adds to the corresponding elements of M, shown graphically in Fig. 1. For 1-foliated symmetries, it is thus possible to realize any $\mathbf{M}$ by stacking 2D SPTs; hence all phases are weak.

On the other hand, for 2- or 3-foliated models, this procedure may not work because $|\psi\rangle_{1 \text {-fol }}$ is not guaranteed to be symmetric under the orthogonal planar symmetries (if it is, we can simply follow the same procedure). Instead, let us define variables $d_{\mathbf{r}}=g_{\mathbf{r}+\mathbf{z}} g_{\mathbf{r}}^{-1}$, which transform under $x y$ planar symmetries but are invariant under all orthogonal symmetries. We may then define nontrivial SSPT wave functions as before, but in terms of $d_{\mathbf{r}}$ instead using the unitary

$$
U=\sum_{\left\{g_{\mathbf{r}}\right\}} f_{2 \mathrm{D}}\left(\left\{d_{\mathbf{r}}\right\}_{r_{z} \in\left\{z_{k}\right\}}\right)\left|\left\{g_{\mathbf{r}}\right\}\right\rangle\left\langle\left\{g_{\mathbf{r}}\right\}\right|,
$$

which is explicitly invariant under the orthogonal symmetries. However, in this case the $\mathbf{M}$ matrix of the 2D SPT does not map directly onto that of the SSPT-instead one should view the 2D SPT as living "in between" the planes of the
SSPT, at $\left\{z_{k}+1 / 2\right\}$. To obtain the $\mathbf{M}$ matrix of the SSPT, one can compute the appropriate type-I and -II cocycles of the 2D SPT in the basis of the $x y$ planar symmetries [63]. This process is shown in Fig. 1. As will be discussed in the next section, unlike for 1-foliated symmetries, there are now allowable phases which cannot be realized by stacking any number of 2D SPTs.

Note that in this discussion we have implicitly ignored nontrivial SSPTs that have trivial $\mathbf{M}$ matrices. Such phases do exist [63]. However, we conjecture that all such phases are weak (they can be realized by stacking 2D linear SSPTs [10]) and therefore irrelevant in the classification of strong phases.

General constraints and invariants. In the presence of orthogonal symmetries, there are general constraints that must be satisfied by M. Conceptually, these arise due to the aforementioned redundancy: the global symmetry $S_{\text {glob }}(g)=$ $\prod_{z} S^{(x y)}(z ; g)=\prod_{x} S^{(y z)}(x ; g)$. Since $y z$ symmetries do not contribute to $\mathbf{M}$, the generator $S_{\text {glob }}(g)$ must therefore manifest trivially in M. This leads to two types of constraints on the elements of $\mathbf{M}$ : The global symmetry must have a trivial type-II cocycle with any other symmetry and a trivial type-I cocycle with itself. We prove that these constraints must hold generally [63]. Let us label the $\alpha$ th generator of $G$ on the $z$ th plane by $i=(\alpha, z)$. Then, the two constraints are expressed as

$$
\sum_{z^{\prime}} M_{(\alpha, z),\left(\beta, z^{\prime}\right)} \equiv 0 \quad \bmod N, \quad \forall \alpha, z, \beta
$$

and

$$
\frac{1}{2} \sum_{z, z^{\prime}} M_{(\alpha, z),\left(\alpha, z^{\prime}\right)} \equiv 0 \quad \bmod N, \quad \forall \alpha .
$$

These constraints define a restricted subgroup of $H^{3}\left[G^{L}, U(1)\right]$ in which 2- or 3-foliated SSPTs must reside. As we will show, there are now allowed phases which cannot be realized by stacking any number of 2D SPTs-these are precisely the strong phases we are searching for. This motivates us to define two types of strong invariants, $F_{1}$ and $F_{2}$, which cannot be changed by stacking with 2D SPTs.

Strong SSPTs: Type 1. Consider $G=\mathbb{Z}_{2 N}$. Then $M_{z z^{\prime}}$ is an $L \times L$ matrix. Pick an arbitrary cut that divides the system into two halves $z<z_{0}$ and $z \geqslant z_{0}$. Then,

$$
F_{1} \equiv \sum_{z<z_{0}} \sum_{z^{\prime} \geqslant z_{0}} M_{z z^{\prime}} \bmod 2
$$

is a $\mathbb{Z}_{2}$-valued global invariant. To see why, view $M_{z z^{\prime}} \bmod 2$ as a $\mathbb{Z}_{2}$ "flux" flowing from vertex $z$ to $z$ ' in the graphical representation. Then, Eq. (3) is a divergence-free constraint at each vertex. The invariant $F_{1}$ is simply the total $\mathbb{Z}_{2}$ flux flowing through a cut at $z_{0}$. It is therefore clear that $F_{1}$ does not depend on the choice of cut $z_{0}$, nor can it be modified by stacking a 2D SPT which amounts to adding closed flux loops locally.

Type 2. Consider $G=\mathbb{Z}_{N} \times \mathbb{Z}_{N}$, so that $M_{(\alpha, z),\left(\beta, z^{\prime}\right)}$ is a $2 L \times 2 L$ matrix. Again pick a cut $z_{0}$. Then,

$$
F_{2} \equiv \sum_{z<z_{0}} \sum_{z^{\prime} \geqslant z_{0}}\left(M_{(1, z),\left(2, z^{\prime}\right)}-M_{(2, z),\left(1, z^{\prime}\right)}\right) \quad \bmod N
$$

is a $\mathbb{Z}_{N}$-valued global invariant. To see how this arises, interpret $M_{(1, z),\left(2, z^{\prime}\right)}$ as a $\mathbb{Z}_{N}$ "flux" flowing from vertex $(1, z)$ to $\left(2, z^{\prime}\right)$. As before, Eq. (3) is a divergence-free constraint on 
this flux and $F_{2}$ measures the total flux flowing across a cut, which therefore does not depend on $z_{0}$ nor can it be modified by stacking with 2D SPTs.

In the Supplemental Material [63], we prove three important statements First, that the invariant $F_{1}$ or $F_{2}$ is the same regardless of whether we consider the $\mathbf{M}$ matrix obtained from $x y$ symmetries or that obtained from $y z$ (or $z x)$ symmetries. Second, 3-foliated systems must have trivial $F_{2}=0$. Third, the set of $F_{1}$ and $F_{2}$ (which we also define for general $G$ ) completely classifies $\mathbf{M}$ modulo stacking with 2D SPTs. Finally, we also provide an explicit construction of a 3-foliated model which realizes a nontrivial type-1 strong phase $F_{1}=1$, and a 2 -foliated model which realizes arbitrary $F_{1}$ and $F_{2}$, thereby demonstrating the existence of such strong phases. Examples of $\mathbf{M}$ matrices with nontrivial $F_{1}$ and $F_{2}$ are shown in Fig. 1 (right).

Let us define a "strong" equivalence relation between SSPTs, under which two phases belong to the same equivalence class if they can be connected with one another by stacking of 2D phases (along with, of course, symmetric local unitary transformations and addition/removal of disentangled degrees of freedom transforming as an on-site linear representation of $G$ [71]). For an arbitrary finite Abelian group $G=\prod_{i} \mathbb{Z}_{N_{i}}$, the set of equivalence classes is given by

$$
\begin{gathered}
C_{3 \text {-fol }}[G]=\prod_{i} \mathbb{Z}_{\mathrm{gcd}\left(2, N_{i}\right)}, \\
C_{2 \text {-fol }}[G]=\prod_{i} \mathbb{Z}_{\mathrm{gcd}\left(2, N_{i}\right)} \times \prod_{i<j} \mathbb{Z}_{\mathrm{gcd}\left(N_{i}, N_{j}\right)},
\end{gathered}
$$

for 3-foliated and 2-foliated models, respectively. The group structure is realized via the stacking operation between two SSPTs. We note that this equivalence relation can be naturally formulated in terms of planar-symmetric local unitary circuits, generalizing the definition of Ref. [10]. Indeed the unitaries $U$ used to construct weak SSPTs are examples of such circuits.

Fracton duals. It is well known that, under a generalized gauge duality $[11,16,29]$, SSPT phases map onto models of fracton topological order [12,58]. The simplest and most wellstudied fracton model is the X-cube model [11], which is obtained by gauging the planar symmetries of the plaquette Ising paramagnet, and hosts fractional quasiparticle excitations with limited mobility including immobile fractons, lineons mobile along lines, and planons mobile within planes (which are either fracton dipoles or lineon dipoles). For our discussion, we will assume that the reader has a rudimentary understanding of the X-cube fracton model and its quasiparticle excitations (see Ref. [34] for a review).

Let us begin with 3-foliated SSPTs, which are dual to "twisted" X-cube fracton topological orders with fractonic charge [58]. The gauge flux $m_{(g, z)}$ of an element $g$ on the plane $z$ is a planon: a composite excitation composed of a lineon antilineon pair on the planes $z+1 / 2$ and $z-1 / 2$, i.e., a lineon dipole. A single lineon can be regarded as a semi-infinite stack of lineon dipoles mobile in the $x$ and $y$ directions. For a more nuanced discussion of the mobility of such excitations, see the Supplemental Material [63].

The constraints on the matrix $\mathbf{M}$ have a simple interpretation in this language: The infinite stack of lineon dipoles, which belongs to the vacuum superselection sector [72], must have trivial braiding statistics with all other lineon dipoles, and a trivial exchange statistic with itself. The invariant $F_{1}$ also has a simple interpretation in this picture: The quantity $e^{2 \pi \mathrm{i} F_{1} / N^{2}}$ corresponds to the braiding (or crossing [46]) statistic of a lineon and its antilineon on the same plane, modulo $e^{4 \pi \mathrm{i} / N^{2}}$.

It is possible to construct fracton topological orders by strongly coupling intersecting stacks of topologically ordered $2 \mathrm{D}$ discrete gauge theories oriented along the $x y, y z$, and $z x$ planes, inducing a type of transition called $p$-string condensation [46,61]. More generally, these stacks of 2D gauge theories can be replaced by arbitrary 1-foliated gauge theories [58]. The twisted X-cube models that emerge from this construction are dual to weak 3-foliated SSPTs constructed via the planar-symmetric local unitaries $U$ in Eq. (2). We walk through this correspondence in more detail in the Supplemental Material [63].

Equivalently, twisted X-cube models dual to weak SSPTs may be obtained by effectively "binding" 2D anyons to existing planons in the fracton model. As an example, consider placing one layer of the doubled semion topological order (with bosonic $e$ and semionic $m$ ) onto a plane $z_{0}$ of the X-cube model, and condensing pairs of $e$ and fracton dipoles in the plane $z_{0}$. The end result is that $x$ or $y$ mobile lineons on plane $z_{0}$ and $m$ become confined, but the bound state of the two remain deconfined and form the new lineon excitations. Since $m$ is a semion, the new lineons now also inherit their semionic statistics. This procedure can be extended to general twisted quantum doubles living on multiple planes $\left\{z_{k}\right\}$, thereby binding more general 2D anyons to the lineons; this process is exactly dual to stacking a 2D SPT according to Eq. (2).

Conversely, strong 3-foliated SSPTs are dual to fracton models that cannot be realized through such a construction. This correspondence sheds light on the $F_{1}$ strong invariant-in $p$-string condensation, lineon crossing statistics are inherited from the self-braiding statistics of fluxes in the 1-foliated gauge theories, and are therefore the square of a flux exchange statistic, i.e., a multiple of $e^{4 \pi i / N^{2}}$ for $G=\mathbb{Z}_{N}$ with $N$ even. In a strong phase, $F_{1}=1$ implies that this statistic is offset by $e^{2 \pi i / N^{2}}$. The fracton dual of the type-1 strong $G=\mathbb{Z}_{2}$ model [63] is an example of such a fracton order in which lineons satisfying a triple fusion rule have a $\pm i$ mutual crossing statistic, and therefore cannot be realized via $p$-string condensation. A Hamiltonian realizing this phase is shown in Fig. 2.

One can also consider the fracton duals of 2 -foliated SSPTs, which are "twisted" versions of the 2-foliated lineonplanon model introduced in Ref. [60]. Furthermore, the Xcube model may be ungauged in two different ways, by regarding either the fracton sector or the lineon sector as the gauge charge. The former procedure results in a paramagnet with $G$-valued degrees of freedom transforming under all three sets of planar symmetries as before, whereas the latter yields a model with two $G$-valued degrees of freedom per site, the first transforming under $x y$ and $y z$ planar symmetries, and the second under $y z$ and $z x$ planar symmetries. The classification of the latter system is given by $\left(C_{2 \text {-fol }}\right)^{2}$. Thus, both type-1 and type- 2 strong SSPTs, as well as arbitrary weak SSPTs, may be constructed. Their fracton duals are variants of the $\mathrm{X}$-cube model whose fracton dipoles exhibit nontrivial braiding and exchange statistics. 


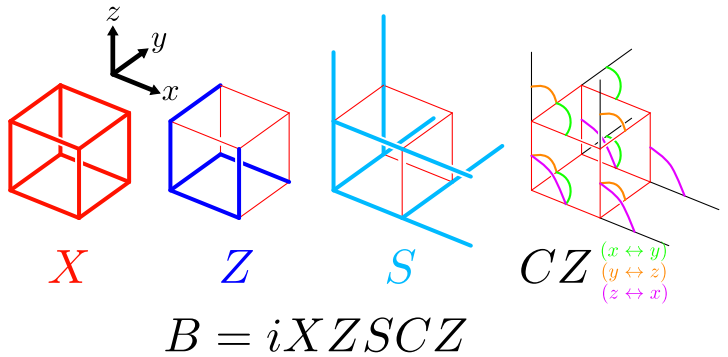

FIG. 2. The commuting (but nonprojector) Hamiltonian describing the fracton dual of the $\mathbb{Z}_{2}$ strong model is shown. Qubit degrees of freedom live on the links. The Hamiltonian is a sum over all cubes $c$ of the term $B_{c}$, shown, which consists of Pauli $X, Z, S=\operatorname{diag}(1, i)$, and $C Z_{12}=(-1)^{\left(Z_{1}-1\right)\left(Z_{2}-1\right) / 4}$ operators between qubits on links of different orientations as shown by the colored lines. In addition, the Hamiltonian also has the usual cross terms $A_{v}^{\mu \nu}$ from the X-cube which is the product of four $Z$ operators lying in the $\mu \nu$ plane touching a vertex $v$. Thus, $H=-\sum_{c} B_{c}-\sum_{v}\left(A_{v}^{x y}+A_{v}^{y z}+A_{v}^{z x}\right)$.

A type-2 strong SSPT can also be diagnosed through the statistical phases of quasiparticles of the gauged dual. Although fractons are immobile particles, we may still define a braiding statistic between two fractons by regarding a single fracton as a semi-infinite stack of fracton dipoles mobile in the $x y$ plane. Consider a $G=\mathbb{Z}_{N} \times \mathbb{Z}_{N}$ model which has two flavors of fractons. Then, let $e^{i \theta_{a b}}$ be the statistical phase obtained by braiding two such fractons of flavors $a$ and $b$ on plane $z_{0}$, where the first index is a semi-infinite stack in the $z \rightarrow \infty$ direction, and the second index in the $z \rightarrow-\infty$ direction. The type- 2 strong invariant is then obtained by $e^{i F_{2} / N}=e^{i N\left(\theta_{a b}-\theta_{b a}\right)}$. This makes it clear why this strong phase with $F_{2} \neq 0$ cannot be obtained by binding $2 \mathrm{D}$ anyons to the fractons, since braiding of $2 \mathrm{D}$ anyons is manifestly symmetric with respect to its two arguments.
Conclusions. We have formulated a classification of strong 3D planar SSPTs. Each phase falls into one of a finite set of equivalence classes modulo stacking with $2 \mathrm{D}$ phases, which we have fully enumerated. For 1-foliated systems, all SSPT phases are weak. For 2-foliated systems, there are two mechanisms by which a phase may be strong, characterized by type1 and type-2 invariants. For 3-foliated systems, only type-1 strong phases exist. Under a generalized gauge duality, our classification has a natural interpretation in terms of $p$-string condensation [46], and we have explicitly constructed strong SSPT models which are dual to fracton phases that cannot be realized via this mechanism. The fractional quasiparticles in these strong phases thus have statistical interactions which cannot be interpreted as the statistics of $2 \mathrm{D}$ anyonic bound states.

There are various natural extensions of our work. A relevant and open question regards the structure of entanglement in strong SSPT phases [25-28,59]. Another is the addition of nontrivial type-III cocycles, which leads to non-Abelian fracton topological orders. Finally, it would be interesting to study the foliation structure of the fracton duals.

Acknowledgments. T.D. thanks Fiona Burnell, Dominic Williamson, Abhinav Prem, and Shivaji Sondhi for many helpful discussions, especially in the early parts of this work. W.S. thanks Xie Chen and Sagar Vijay for helpful discussions. T.D. acknowledges support from the Charlotte Elizabeth Procter Fellowship at Princeton University. W.S. is supported by the National Science Foundation under Award No. DMR-1654340 and the Institute for Quantum Information and Matter at Caltech. J.W. was supported by NSF Grant No. PHY-1606531 and Institute for Advanced Study. This work is also supported by NSF Grant No. DMS-1607871 "Analysis, Geometry and Mathematical Physics" and the Center for Mathematical Sciences and Applications at Harvard University.
[1] D. Gaiotto, A. Kapustin, N. Seiberg, and B. Willett, J. High Energy Phys. 02 (2015) 172.

[2] C. D. Batista and Z. Nussinov, Phys. Rev. B 72, 045137 (2005).

[3] Z. Nussinov and G. Ortiz, Proc. Natl. Acad. Sci. USA 106, 16944 (2009).

[4] Z. Nussinov and G. Ortiz, Ann. Phys. 324, 977 (2009).

[5] X. Chen, Z.-X. Liu, and X.-G. Wen, Phys. Rev. B 84, 235141 (2011).

[6] X. Chen, Z.-C. Gu, Z.-X. Liu, and X.-G. Wen, Science 338, 1604 (2012)

[7] X. Chen, Z.-C. Gu, Z.-X. Liu, and X.-G. Wen, Phys. Rev. B 87, 155114 (2013).

[8] T. Senthil, Annu. Rev. Condens. Matter Phys. 6, 299 (2015).

[9] Y. You, T. Devakul, F. J. Burnell, and S. L. Sondhi, Phys. Rev. B 98, 035112 (2018).

[10] T. Devakul, D. J. Williamson, and Y. You, Phys. Rev. B 98, 235121 (2018)

[11] S. Vijay, J. Haah, and L. Fu, Phys. Rev. B 94, 235157 (2016).
[12] Y. You, T. Devakul, F. J. Burnell, and S. L. Sondhi, arXiv: 1805.09800.

[13] B. Yoshida, Phys. Rev. B 88, 125122 (2013).

[14] T. Devakul, Y. You, F. J. Burnell, and S. L. Sondhi, SciPost Phys. 6, 007 (2019).

[15] T. Devakul, Phys. Rev. B 99, 235131 (2019).

[16] D. J. Williamson, Phys. Rev. B 94, 155128 (2016).

[17] M. E. J. Newman and C. Moore, Phys. Rev. E 60, 5068 (1999).

[18] C. Castelnovo and C. Chamon, Philos. Mag. 92, 304 (2012).

[19] R. Raussendorf and H. J. Briegel, Phys. Rev. Lett. 86, 5188 (2001).

[20] D. V. Else, S. D. Bartlett, and A. C. Doherty, New J. Phys. 14, 113016 (2012).

[21] R. Raussendorf, C. Okay, D.-S. Wang, D. T. Stephen, and H. P. Nautrup, Phys. Rev. Lett. 122, 090501 (2019).

[22] D. T. Stephen, H. P. Nautrup, J. Bermejo-Vega, J. Eisert, and R. Raussendorf, Quantum 3, 142 (2019).

[23] T. Devakul and D. J. Williamson, Phys. Rev. A 98, 022332 (2018). 
[24] A. K. Daniel, R. N. Alexander, and A. Miyake, Quantum 4, 228 (2020).

[25] D. J. Williamson, A. Dua, and M. Cheng, Phys. Rev. Lett. 122, 140506 (2019).

[26] A. T. Schmitz, S.-J. Huang, and A. Prem, Phys. Rev. B 99, 205109 (2019).

[27] D. T. Stephen, H. Dreyer, M. Iqbal, and N. Schuch, Phys. Rev. B 100, 115112 (2019).

[28] L. Zou and J. Haah, Phys. Rev. B 94, 075151 (2016).

[29] W. Shirley, K. Slagle, and X. Chen, SciPost Phys. 6, 041 (2019).

[30] C. Chamon, Phys. Rev. Lett. 94, 040402 (2005).

[31] S. Bravyi, B. Leemhuis, and B. Terhal, Ann. Phys. 326, 839 (2011).

[32] J. Haah, Phys. Rev. A 83, 042330 (2011).

[33] S. Vijay, J. Haah, and L. Fu, Phys. Rev. B 92, 235136 (2015).

[34] R. M. Nandkishore and M. Hermele, Annu. Rev. Condens. Matter Phys. 10, 295 (2019).

[35] S. Pai, M. Pretko, and R. M. Nandkishore, Phys. Rev. X 9 , 021003 (2019).

[36] M. Pretko and L. Radzihovsky, Phys. Rev. Lett. 120, 195301 (2018).

[37] M. Pretko and L. Radzihovsky, Phys. Rev. Lett. 121, 235301 (2018).

[38] L. Radzihovsky and M. Hermele, Phys. Rev. Lett. 124, 050402 (2020).

[39] A. Prem, S.-J. Huang, H. Song, and M. Hermele, Phys. Rev. X 9, 021010 (2019).

[40] A. Prem and D. J. Williamson, SciPost Phys. 7, 068 (2019).

[41] M. Pretko, Phys. Rev. B 95, 115139 (2017).

[42] M. Pretko, Phys. Rev. B 96, 035119 (2017).

[43] A. Prem, M. Pretko, and R. M. Nandkishore, Phys. Rev. B 97, 085116 (2018).

[44] D. Bulmash and M. Barkeshli, Phys. Rev. B 100, 155146 (2019).

[45] K. Slagle and Y. B. Kim, Phys. Rev. B 97, 165106 (2018).

[46] H. Ma, E. Lake, X. Chen, and M. Hermele, Phys. Rev. B 95, 245126 (2017).

[47] Y. You, D. Litinski, and F. von Oppen, Phys. Rev. B 100, 054513 (2019).
[48] H. Song, A. Prem, S.-J. Huang, and M. A. Martin-Delgado, Phys. Rev. B 99, 155118 (2019).

[49] D. A. Johnston, J. Phys. A 45, 405001 (2012).

[50] C. Castelnovo, C. Chamon, and D. Sherrington, Phys. Rev. B 81, 184303 (2010)

[51] A. Gromov, Phys. Rev. X 9, 031035 (2019).

[52] A. Dua, I. H. Kim, M. Cheng, and D. J. Williamson, Phys. Rev. B 100, 155137 (2019).

[53] S. Pai and M. Hermele, Phys. Rev. B 100, 195136 (2019).

[54] W. Shirley, K. Slagle, Z. Wang, and X. Chen, Phys. Rev. X 8, 031051 (2018).

[55] W. Shirley, K. Slagle, and X. Chen, Phys. Rev. B 99, 115123 (2019).

[56] W. Shirley, K. Slagle, and X. Chen, SciPost Phys. 6, 015 (2019).

[57] T. Wang, W. Shirley, and X. Chen, Phys. Rev. B 100, 085127 (2019).

[58] W. Shirley, K. Slagle, and X. Chen, arXiv:1907.09048.

[59] A. Dua, P. Sarkar, D. J. Williamson, and M. Cheng, arXiv: 1909.12304.

[60] W. Shirley, K. Slagle, and X. Chen, Ann. Phys. 410, 167922 (2019).

[61] S. Vijay, arXiv:1701.00762.

[62] M. Levin and Z.-C. Gu, Phys. Rev. B 86, 115109 (2012).

[63] See Supplemental Material at http://link.aps.org/supplemental/ 10.1103/PhysRevResearch.2.012059 for a review of the group cohomological classification, discussions of various technical details, proofs of statements from the main text, and explicit construction of strong models.

[64] D. V. Else and C. Nayak, Phys. Rev. B 90, 235137 (2014).

[65] M. de Wild Propitius, arXiv:hep-th/9511195.

[66] J. C. Wang and X.-G. Wen, Phys. Rev. B 91, 035134 (2015).

[67] X.-G. Wen and A. Zee, Phys. Rev. B 46, 2290 (1992).

[68] X.-G. Wen, Nat. Sci. Rev. 3, 68 (2016).

[69] J. C. Wang, L. H. Santos, and X.-G. Wen, Phys. Rev. B 91, 195134 (2015).

[70] J. C. Wang, Z.-C. Gu, and X.-G. Wen, Phys. Rev. Lett. 114, 031601 (2015)

[71] X. Chen, Z.-C. Gu, and X.-G. Wen, Phys. Rev. B 82, 155138 (2010).

[72] A. Kitaev, Ann. Phys. 321, 2 (2006) 